Short Paper

\title{
Occurrence of an Unusual Accessory Growth Center on the Statolith of the Japanese Common Squid Todarodes pacificus (Cephalopoda: Ommastrephidae)
}

\author{
Yuzuru Ikeda, ${ }^{* 1, \dagger}$ Nobuaki Arai, ${ }^{* 1, \dagger \dagger}$ Wataru Sakamoto, ${ }^{{ }_{1} 1}$ and Tatsuro Murayama ${ }^{* 2}$ \\ ${ }^{*}$ Division of Applied Biosciences, Graduate School of Agriculture, Kyoto University, \\ Kyoto 606-8502, Japan \\ *2Shimane Prefectural Fisheries Experimental Station, Setogashima, \\ Hamada, Shimane 697-0051, Japan
}

(Received March 9, 1998)

Key words: Statolith, Accessory growth center, microincrement, Todarodes pacificus

During a survey of statoliths from the Japanese common squid Todarodes pacificus to reconstruct the environmental variables of habitat (e.g., Ikeda et $\left.a l .{ }^{1}\right)$, we obtained an unusual statolith with an accessory growth center. In this note, we describe the features of that statolith.

An unusual statolith was obtained from a mature male T. pacificus of $243 \mathrm{~mm}$ dorsal mantle length (DML) and $283 \mathrm{~g}$ body weight (BW), which was caught by jigging from the Sea of Japan in the vicinity of Tottori Prefecture $\left(40^{\circ} 10^{\prime} \mathrm{N}, 132^{\circ} 50^{\prime} \mathrm{E}\right)$ on 1 September 1988 . For control, we observed statoliths of thirty-four male $T$. pacificus (DML $176-259 \mathrm{~mm}, \mathrm{BW} 110-401 \mathrm{~g}$, immature-mature judged by the maturity scale of Ikeda et al. ${ }^{2)}$ ) caught together with the unusual specimen.

Figure 1 shows the light microphotographs of the left statolith of the unusual specimen. The supposed accessory growth center (AGC) is situated on the dorsal dome near its boundary to the wing (Fig. 1A). The body of AGC is round in shape, which seemed to be consisted with two parts, i) wedge like structure originated from the wing, covered on the tip of the dorsal dome, and ii) scalloped structure above the wedge like structure (Fig. 1B). The long axis of the AGC body parallel to the dorsal line of the main statolith body and short axis of the AGC body parallel to the frontal line of the main statolith body (terminology after Lipinski et al. ${ }^{3)}$ ) was $260 \mu \mathrm{m}$ and $210 \mu \mathrm{m}$, respectively. Inside the AGC, the microincrements were observed (Fig. 1C). Those microincrements seemed to originate from the base of the AGC at the dorsal dome of the statolith and deposit peripherally. Counting of microincrements was made on a polished statolith four times, and the average of the middle two values obtained from the four counts was used as the microincrement number. The microincrement number in the AGC was 64 . The width of microincrement in the AGC, which was measured by micrometer attached to a light microscope was ca $2.5 \mu \mathrm{m}$. At the peripheral portion of the $\mathrm{AGC}$, two particularly dark rings (like check) were visible (Fig. 1C).
Figure 2 shows the dimensions of the unusual left statolith with the AGC and the statoliths of the control males. The following dimensions ${ }^{4)}$ were investigated: TSL, total statolith length; LDL, lateral plus dorsal dome length; DWR, dorsal wing to tip of rostrum; WSL, wing length. The statolith of $T$. pacificus showed linear growth in relation with the body size and each dimension (Fig. 2). Those dimensions of the unusual left statolith with the AGC were $1075 \mu \mathrm{m}$ in TSL, $825 \mu \mathrm{m}$ in LDL, $1100 \mu \mathrm{m}$ in DWR, and $1100 \mu \mathrm{m}$ in WSL. Comparing with the control, the wing of the unusual left statolith with the AGC is relatively elongated (Fig. 2B, D). This would suggest that the occurrence of the wedge structure of the AGC relates with elongate wing of the main statolith body (see Fig. 1B). The microincrement number of the left statolith with the AGC of the unusual specimen, corresponding with age of an individual, ${ }^{5)}$ was 276 (days). Those values of five mature control specimens with similar DML (231-255 mm) and TSL $(1075-1325 \mu \mathrm{m})$ to the unusual specimen were 263,277 , 287,294 , and 308 (days). Thus, statolith growth of the unusual specimen, except for the elongate wing, appears to be as normal as control males.

The right statolith in this unusual specimen had an AGC in the same location, i.e., on the dorsal dome near its boundary to the wing. However, this is consisted only with the wedge like structure with no visible microincrements inside. The size and dimensions of that AGC were, long axis $90 \mu \mathrm{m}$, short axis $85 \mu \mathrm{m}$, TSL $1025 \mu \mathrm{m}$, LDL $700 \mu \mathrm{m}$, DWR $1025 \mu \mathrm{m}$, and WSL $1075 \mu \mathrm{m}$, which also indicates, similar to the left statolith, elongation of the wing. Microincrement number of this right statolith were 273 . In Pleuronectids fish, accessory growth centers are observed inside the main body of the otolith and this was found to occur at the time of metamorphosis. ${ }^{6)}$ In the case of squids, AGC was observed outside of the statolith main body (present study). This difference in location of the AGC between fish and squid might relate with the fact that squid does not exhibit metamorphosis. Another reason must be at work for the occurrence of the AGC in squid 


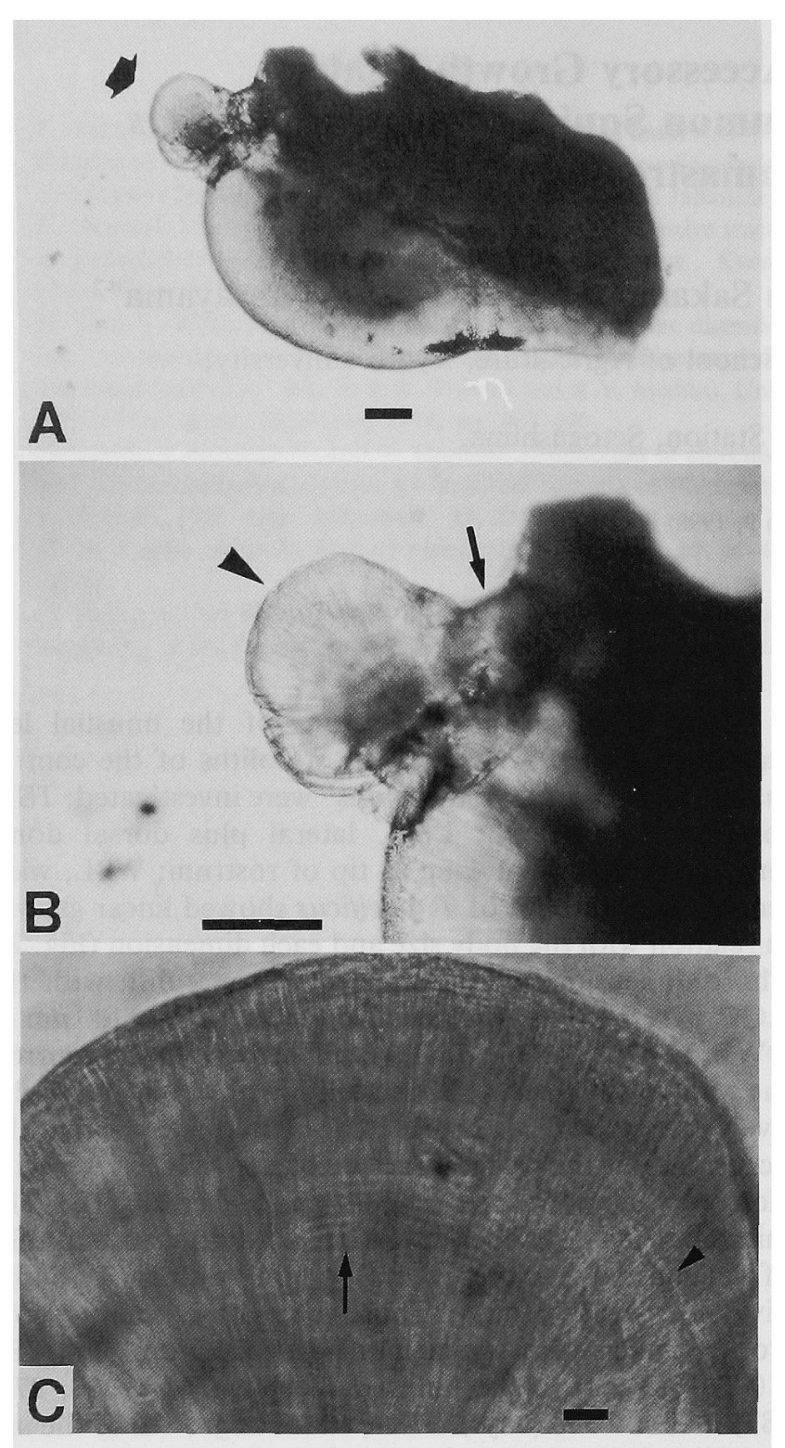

Fig. 1. Microphotographs of the unusual left statolith with an accessory growth center (AGC), which was obtained from a mature male Todarodes pacificus with DML $243 \mathrm{~mm}$ and BW $283 \mathrm{~g}$.

(A) Whole structure of the unusual statolith with AGC (arrow), (B) The AGC. Arrow and arrow head indicate wedge like structure and scalloped structure of the AGC, respectively. Note, the AGC originates from wing of statolith main body, (C) Microincrements (arrow) and check like structures (arrow head) in the AGC. Scale bar, $100 \mu \mathrm{m}$ for $\mathrm{A}$ and $\mathrm{B}, 10 \mu \mathrm{m}$ for $\mathrm{C}$.

statolith, especially the elongation of the statolith wing. Further investigations such on the developmental process of the AGC and on deposition periodicity of microincrements in the AGC are necessary.

We acknowledge R. Kuranaga for his cooperation of squid collection, H. Kidokoro for his assistance with microincrement count, and J. C. Hunt for his critical reading of the manuscript. This study was supported in part by a Grant-in-Aid for Encouragement of Young Scientists, the Ministry of Education, Science, Sports and Culture of Japan.

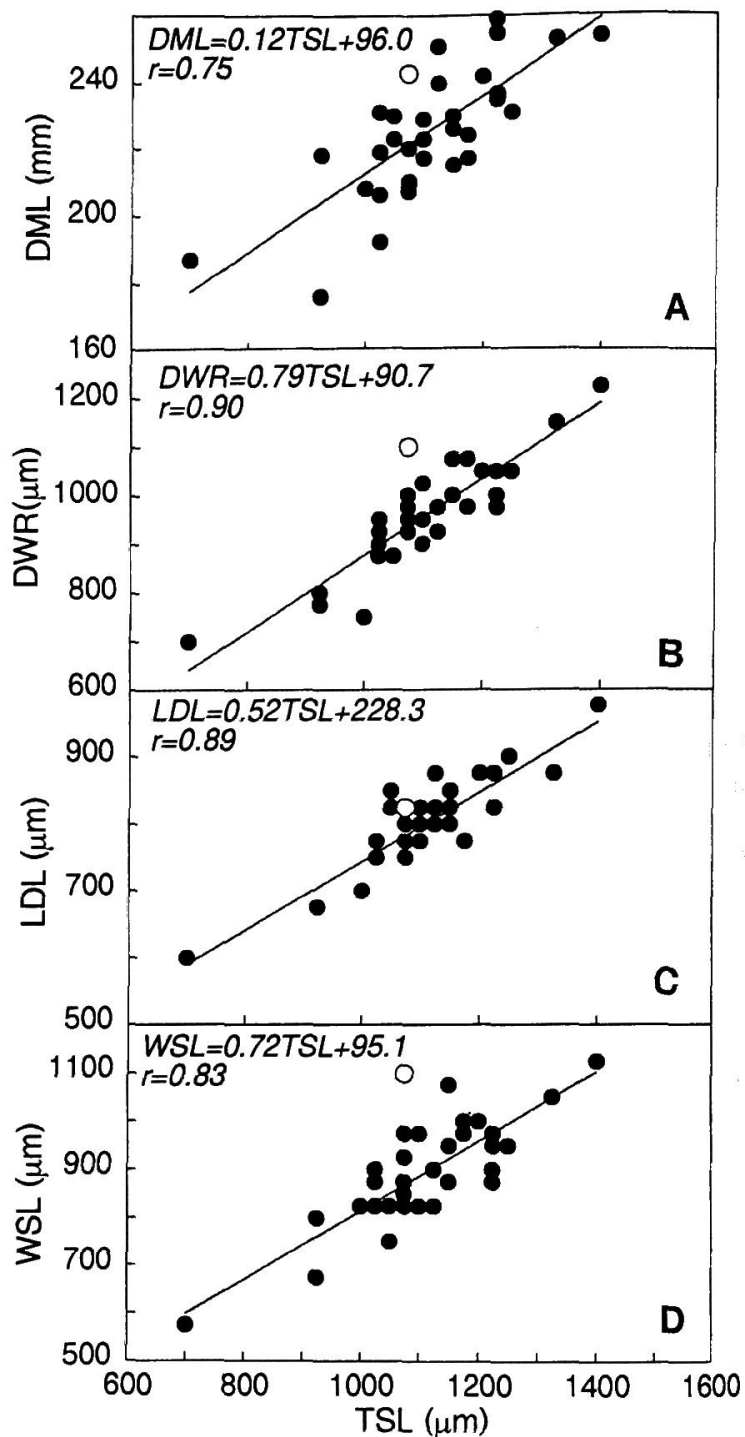

Fig. 2. Comparison of statolith dimensions for the unusual left statolith $(O)$ and the statoliths of control males ( $\bullet$ ) (DML 176-259 $\mathrm{mm}$, BW 110-401 g).

Relationship between (A) total statolith length (TSL) and DML, (B) TSL and dorsal wing to tip of rostrum (DWR), (C) TSL and lateral plus dorsal dome length (LDL), and (D) TSL and wing length (WSL).

Line represents a regression line.

\section{References}

1) Y. Ikeda, N. Arai, W. Sakamoto, J. Adachi, T. Murayama, K. Maeda, and K. Yoshida: Fisheries Sci., 63, 325-326 (1997).

2) Y. Ikeda, Y. Sakurai, and K. Shimazaki: Nippon Suisan Gakkaishi, 57, 2237-2241(1991).

3) M. Lipiński, E. Dawe, and Y. Natsukari: in "Squid Age Determination Using Statolith" (eds. by P. Jereb., S. Rogonese, and S. von Boletzky), N. T. R.-I. T. P. P., Special Pub., 1, 1991, pp.77-81.

4) M. R. Lipinski and M. D. Durholtz: Antarctic Sci., 6, 215-222 (1994).

5) Y. Nakamura and Y. Sakurai: Nippon Suisan Gakkaishi, 57, 20072011 (1991).

6) A. Jearld Jr., S. L. Sass, and M. F. Davis: Fish. Bull. U.S., 91, 6575 (1993). 